\title{
A PHRAGMÉN-LINDELÖF TYPE THEOREM FOR A CERTAIN CLASS OF GENERALIZED SUBHARMONIC FUNCTIONS
}

TORBJÖRN HEDBERG

We shall consider a class of functions introduced by Y. Domar [1]. The class contains the positive subharmonic functions and is defined as follows:

Let $E$ be an open connected subset of the $n$-dimensional Euclidean space $\mathrm{R}^{n}$ and suppose that $B \geqq 1$ is a given number. The function $u$ is then said to belong to the class $S(B)$ in $E$ if it satisfies the following conditions:

(i) $u$ is non-negative and measurable

(ii) $u$ is bounded on every compact subset of $E$

(iii) for every $n$-dimensional sphere $S_{R}\left(x_{0}\right) \subset E$ with centre $x_{0}$ and radius $R$ we have

$$
u\left(x_{0}\right) \leqq \frac{B}{S_{R}} \int_{S_{R}\left(x_{0}\right)} u d x=B A\left(x_{0}, u, R\right),
$$

where $S_{R}$ denotes the volume of the sphere.

In this paper we always take $n \geqq 2$ and $E=\mathrm{R}^{n}$ and we denote the points by $x=\left(x^{1}, x^{2}, \ldots, x^{n}\right)$ and we also write $|x|=\left(\left(x^{1}\right)^{2}+\ldots+\left(x^{n}\right)^{2}\right)^{\frac{1}{2}}$.

The problem to be studied here consists in finding conditions on a function $v$ such that if $u \leqq v$ and $u \in S(B)$ then $u=0$.

The functions $v$ that we are going to consider vanish outside a proper subset of $E$. In particular we shall in the case $E=\mathrm{R}^{2}$ study a function $v$ vanishing outside a sector of the plane with its centre at the origin. This will give us a generalization of the well-known Phragmén-Lindelöf theorem for subharmonic functions (see e.g. [3, p. 44]).

We start by proving a theorem concerning functions $v$ satisfying a more general condition. Similar but sharper results follow in the case $B=1$ from the estimates of harmonic measures in [2]. 
Let $D$ be a region in $\mathrm{R}^{n}$. We shall use the following definitions:

$$
\begin{aligned}
\theta_{\xi} & =\left\{x \mid x \in D \text { and } x^{1}=\xi\right\}, \\
\theta(\xi) & =m \theta_{\xi},
\end{aligned}
$$

where $m$ denotes the $(n-1)$-dimensional Lebesgue measure.

We put $p=(n-1)^{-1}$.

TheOREM 1. Suppose that the region $D$ satisfies the following conditions:

(i) $\theta_{\xi}=\varnothing$ for $\xi<0$ and $\theta(\xi)>0$ for $\xi \geqq 0$;

(ii) there exists some monotone positive increasing function $h$ on $\mathbf{R}_{+}$, such that $h(t)$ tends to 1 as $t$ tends to 0 , and such that

$$
\left[h\left(\frac{\left|\xi-\xi_{0}\right|}{\theta\left(\xi_{0}\right)^{p}}\right)\right]^{-1} \leqq \frac{\theta(\xi)}{\theta\left(\xi_{0}\right)} \leqq h\left(\frac{\left|\xi-\xi_{0}\right|}{\theta\left(\xi_{0}\right)^{p}}\right)
$$

for all $\xi$ and $\xi_{0}>0$ such that $\left|\xi-\xi_{0}\right| \leqq 2 \theta\left(\xi_{0}\right)^{p}$.

For each $B$ not less than 1 but smaller than some constant only depending on $h$ and greater than 1 we can then choose $\lambda>0$ such that, if

(iii) $u \in S(B)$ in $\mathrm{R}^{n}$,

(iv) $u \leqq \exp \left(\lambda \int_{0}^{x 1} \theta^{-p} d t\right)$ for $x$ in $D$

(v) $u=0$ for $x$ not in $D$,

then $u=0$ everywhere.

Proof. Suppose that $u$ satisfies the above conditions and let $v$ be the function defined by the right hand side members of (iv) and (v). Let $x_{0}$ be an arbitrary point in $D$. For every $B \geqq 1$,

$$
\begin{aligned}
B A\left(x_{0}, v, R\right) & =v\left(x_{0}\right) \frac{B}{S_{R}} \int_{S_{R}\left(x_{0}\right) \cap D} \exp \left(\lambda \int_{x_{0} 1}^{x^{1}} \theta^{-p} d t\right) d x \\
& \leqq B v\left(x_{0}\right) \frac{m\left(S_{R}\left(x_{0}\right) \cap D\right)}{S_{R}} \exp \left(\lambda R \sup \theta(t)^{-p}\right),
\end{aligned}
$$

where the supremum is taken over all $t$ such that $\left|t-x_{0}{ }^{1}\right| \leqq R$. We then take $R=2 \theta\left(x_{0}^{1}\right)^{p}$. It follows immediately from condition (ii) that

$$
\theta\left(x^{1}\right) \geqq \theta\left(x_{0}^{1}\right) h(2)^{-1} \quad \text { if }\left|x_{0}^{1}-x^{1}\right| \leqq R .
$$


Denote the intersection of the set $\left\{x|| x_{0}{ }^{1}-x^{1} \mid \leqq \delta R\right\}$ with $S_{R}\left(x_{0}\right)$ and with $D$ by $S_{R}{ }^{\delta}\left(x_{0}\right)$ and $D^{\delta}$, respectively. The condition (ii) then gives us that the measure of the set $D^{\delta}$ satisfies

$$
m D^{\delta}=2 R \delta \theta\left(x_{0}{ }^{1}\right)\left(1+q_{1}(\delta)\right)=R^{n} 2^{2-n} \delta\left(1+q_{1}(\delta)\right),
$$

where $q_{1}$ tends to 0 uniformly in $x_{0}$ as $\delta$ tends to 0 . Furthermore we have that

$$
m S_{R}^{\delta}\left(x_{0}\right)=2 R^{n} \delta \omega_{n-1}\left(1+q_{2}(\delta)\right),
$$

where $q_{2}$ tends to zero with $\delta$ and where $\omega_{n-1}$ is the volume of the $(n-1)$ dimensional unit ball.

This shows that if $\delta>0$ is chosen small enough we can find an $\varepsilon>0$ such that the measure of the part of $S_{R}{ }^{\delta}\left(x_{0}\right)$ not in $D^{\delta}$ is larger than $\varepsilon R^{n}$ and we have proved the existence of a $C<1$ such that the following relation holds:

$$
m\left(S_{R}\left(x_{0}\right) \cap D\right)<C S_{R} \quad \text { for all } x_{0} \text { in } D .
$$

We can now prove the theorem for a coefficient $B$ such that $1 \leqq B<1 / C$. We introduce numbers $B^{\prime}>B$ and $d$ such that $B^{\prime} C<d<1$ and choose $\lambda>0$ such that

$$
\exp \left(2 \lambda h(2)^{p}\right)<d^{-1}
$$

Since $B^{\prime}<d / C$, (1)-(4) show that for every $x \in D$ there is an $R$ such that

Put

$$
B^{\prime} A(x, v, R)<v(x) \text {. }
$$

$$
M=\sup _{x} u(x) / v(x)<\infty .
$$

If $u$ were not identically vanishing there would exist an $x_{0} \in D$ such that

$$
u\left(x_{0}\right)>B B^{\prime-1} M v\left(x_{0}\right) \text {. }
$$

But on the other hand

$$
u\left(x_{0}\right) \leqq B A\left(x_{0}, u, R\right) \leqq M B A\left(x_{0}, v, R\right)<B B^{\prime-1} M v\left(x_{0}\right)
$$

for some $R$ chosen as above.

This contradiction proves the theorem.

Next we shall apply this theorem to a special region in the plane. For simplicity we now denote the points by $z=(x, y)$ and put $\arg z=\varphi$ and $|z|=r$. The region we shall consider is the region between two straight half-lines forming an angle $\beta$ at the origin, $0<\beta<2 \pi$, and we denote as before this region by $D$. 
If we apply our theorem we find that if $B_{0}>1$ is small enough then for every $B, 1 \leqq B \leqq B_{0}$, there exists a number $\alpha>0$ such that, if

(i) $u \in S(B)$ in $\mathrm{R}^{2}$,

(ii) $u=O\left(r^{\alpha}\right)$ uniformly in $D$ for $r$ tending to infinity,

(iii) $u=0$ for $z$ not in $D$,

then $u=0$.

We assume that $B$ is sufficiently close to 1 to guarantee the existence of one such $\alpha$ and we denote by $\alpha(B, \beta)$ the least upper bound of all such $\alpha$.

We want to prove that $\alpha(B, \beta)$ is close to the corresponding value for subharmonic functions if $B$ is close to 1 . Before we do this we state without proof a trivial lemma, where $D$ is as above, i.e. with angle $\beta$. We also suppose that the region is symmetric with respect to the positive $x$-axis.

LEMma. Let $\beta<\beta_{2}<2 \pi$ and $\delta>0$ be arbitrary and let

$$
v(z)=\max \left(r^{\pi / \beta_{2}-\delta / 2} \cos \pi \varphi / \beta_{2}, 0\right) .
$$

Then there exists a number $B>1$ such that for every $z \in D$ the inequality $v(z)>B A(z, v, R)$ holds for some $R$.

We can now prove our second theorem.

Theorem 2. $\lim _{B \rightarrow 1} \alpha(B, \beta)=\alpha(1, \beta)=\pi / \beta$ for every $\beta, 0<\beta<2 \pi$.

Proof. The Phragmén-Lindelöf theorem for subharmonic functions $\left[3\right.$, p. 44] gives us that $\alpha(1, \beta)=\pi / \beta$. Let $\beta<\beta_{1}<\beta_{2}<2 \pi$ and let $D$ and $D_{1}$ be regions of the above type, symmetric around the positive $x$-axis and with opening angles $\beta$ and $\beta_{1}$ respectively. Assume moreover that the vertices are $(0,0)$ and $(-1,0)$ respectively. Let $\delta$ be a given number and choose $v$ and $B$ according to the lemma. Suppose that $u$ is a function which satisfies

(i) $u \in S(B)$ in $\mathrm{R}^{2}$,

(ii) $u=O\left(r^{\pi / \beta_{2}-\delta}\right)$ uniformly in $D$ for $r$ tending to infinity,

(iii) $u=0$ for $z$ not in $D$.

We can assume that $u$ is continuous for if not we could reduce the problem by taking a convolution of $u$ with some suitable function and then consider a slightly different region. Put

$$
M=\sup _{D} \frac{u(x, y)}{v(x+1, y)}<\infty .
$$


This supremum is attained at some finite point $z_{0}=\left(x_{0}, y_{0}\right) \in D$. If $M>0$, then we have by the lemma that, for some $R$,

$$
\begin{aligned}
u\left(z_{0}\right)=M v\left(x_{0}+1, y_{0}\right) & >M B A\left(\left(x_{0}+1, y_{0}\right), v, R\right) \\
& \geqq B A\left(\left(x_{0}, y_{0}\right), u, R\right) \geqq u\left(z_{0}\right) .
\end{aligned}
$$

The contradiction proves that $u=0$ which implies that $\alpha(B, \beta) \geqq \pi / \beta_{2}-\delta$. Since $\alpha(B, \beta)$ is non-increasing as a function of $B$ and since $\delta$ and $\pi / \beta-\pi / \beta_{2}$ can be chosen arbitrarily small the theorem follows.

\section{REFERENCES}

1. Y. Domar, On the existence of a largest subharmonic minorant of a given function, Ark. Mat. 3 (1957), 429-440.

2. K. Haliste, Estimates of harmonic measures, Ark. Mat. 6 (1965), 1-37.

3. R. Nevanlinna, Eindeutige analytische Funktionen (Grundlehren math. Wiss. 46), Springer-Verlag, Berlin, 1936. 